\title{
Amnios et problèmes de surface oculaire
}

Claude J. Giasson, Cindy Bouchard, Hélène Boisjoly, Lucie Germain

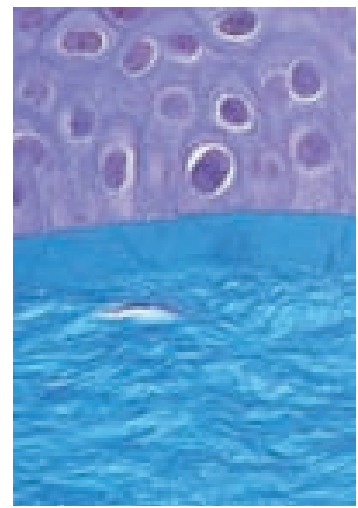

favoriserait la prolifération de cellules moins différenciées, aptes à reconstruire l'épithélium cornéen. La membrane amniotique contient des cytokines, réduit l'acuité des réactions immunologiques et possède des propriétés antalgiques, anti-bactériennes et anti-inflammatoires; de plus, elle favorise, comme le fait la peau fœtale, une guérison avec un minimum de cicatrices. La connaissance des mécanismes d'action de la membrane amniotique obtenue grâce à la recherche pourrait fournir de nouvelles avenues pharmacologiques afin de traiter des maladies de la surface oculaire. <
La membrane amniotique est prélevée à la naissance d'un enfant normal par césarienne facultative auprès de donneuses séronégatives pour le VIH, l'hépatite $B$ et $C$ et la syphilis. Dans des conditions qui en assurent la stérilité, l'amnios est rincé, séparé du chorion, étalé sur un support de nitrocellulose et coupé en petits rectangles qui sont congelés à $-80^{\circ} \mathrm{C}$ ou déshydratés et stérilisés. La congélation dévitalise l'épithélium amniotique et la déshydratation débarrasse le tissu des composantes cellulaires. Puisque la membrane basale amniotique favorise la migration des cellules conjonctivales ou cornéennes, le stroma amniotique est souvent appliqué contre le stroma cornéen ou épiscléral [1]. Dans l'orientation contraire, le stroma amniotique réduit l'inflammation aiguë [2], en captant les cellules inflammatoires, avant d'être digéré et de disparaître [3].
C.J. Giasson : École d'Optométrie, Université de Montréal, CP 6128, Succursale Centre-ville, Montréal (Québec), JlH 5N4 Canada. Laboratoire d'Organogenèse Expérimentale (LOEX), CHA Pavillon Saint-Sacrement. Unité de recherche en santé de la vision, Hôpital Maisonneuve-Rosemont, Montréal (Québec), J1H 5N4 Canada. Département

Biologie de l'amnios et de la surface oculaire

Comme la cornée, la membrane amniotique est avasculaire. Elle obtient nutriment et oxygène du liquide chorionique et amniotique, ainsi que des vaisseaux de la surface fœtale [1]. La monocouche de cellules épithéliales cubiques s'appuie sur une épaisse membrane basale qui fait place à un stroma acellulaire de tissu conjonctif compact, puis à un stroma, peuplé de fibroblastes (Figure I). Enfin, une couche spongieuse fibrillaire et réticulée sépare l'amnios et le chorion [3].

d'ORL-ophtalmologie, Faculté de Médecine, Université Laval, Québec (Québec), J1H 5N4 Canada.

C. Bouchard : École d'Optométrie, Université de Montréal, CP 6128, Succursale Centre-ville, Montréal (Québec), J1H 5N4 Canada.

H. Boisjoly: Unité de recherche en santé de la vision,

Hôpital Maisonneuve-Rosemont, Montréal (Québec),

J1H 5N4 Canada.

Département d'ophtalmologie, Faculté de Médecine, Université de Montréal, Montréal (Québec), JIH 5N4 Canada.

L. Germain :

Laboratoire d'organogenèse expérimentale ( $L O E X)$, CHA Pavillon Saint-Sacrement, Département d'ORL-ophtalmologie, Faculté de Médecine, Université Laval, Québec (Québec), JIH 5N4 Canada. giassonc@opto.umontreal.ca

Article reçu le 27 avril 2005, accepté le 21 mars 2006. 
La surface antérieure de l'œil contient deux populations de cellules souches adultes: des cellules du cul-de-sac conjonctival formant les cellules épithéliales et les cellules en gobelet, ainsi que des cellules du limbe cornéen (Figure 2). Cette zone de transition où la cornée devient transparente est le siège de ces cellules qui constituent l'unique source pour la régénération normale de tout l'épithélium

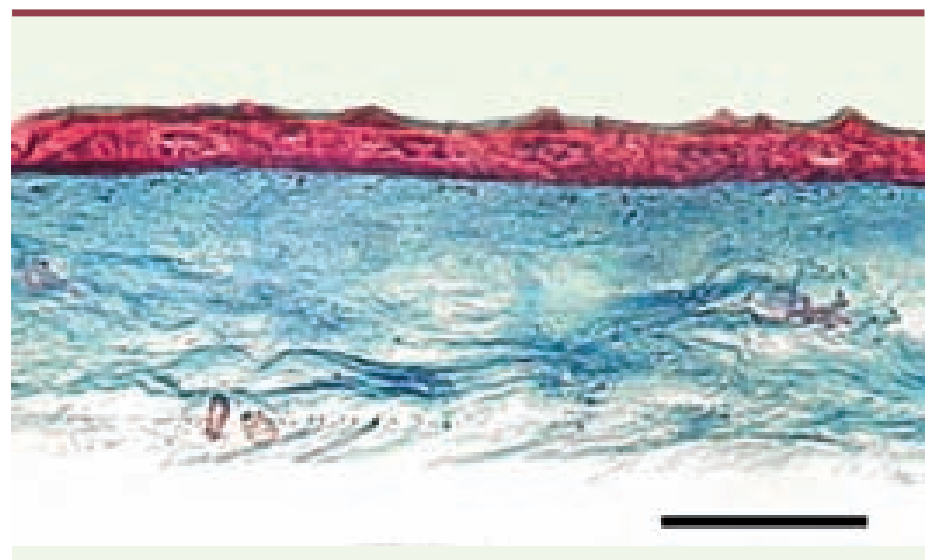

Figure 1. Coupe histologique d'une membrane amniotique (colorée au trichrome de Masson, courtoisie de M. Alexandre Deschambeault).

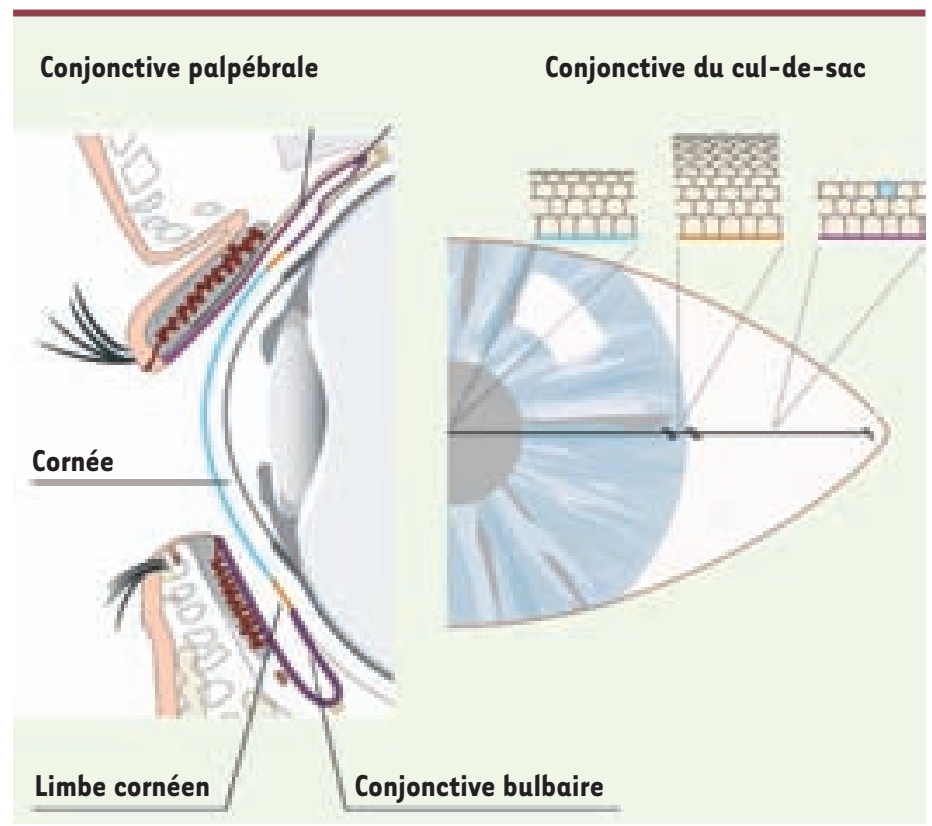

Figure 2. Section sagittale au centre du globe oculaire, montrant les paupières, les portions palpébrale, bulbaire et du cul-de-sac de la conjonctive ainsi que la cornée. L'arrangement histologique de la couche épithéliale de la cornée, du limbe cornéen et de la conjonctive bulbaire est représenté selon un code de couleur illustré dans la vue de face à droite. Cette vue de face illustre les mêmes structures et les types d'épithélium stratifié squameux non kératinisé de la surface oculaire: épithélium de la cornée (bleu), épithélium limbique (orangé) et épithélium de la conjonctive bulbaire (violet). Une cellule à gobelet est représentée en bleu sur le dessin de la conjonctive bulbaire. cornéen durant toute la vie de l'individu. Contrairement aux cellules de l'épithélium cornéen, les cellules limbiques ont un très grand potentiel de prolifération en culture, mais se divisent peu fréquemment in vivo. Ces observations ont été déterminantes lorsque le limbe a été proposé comme site des cellules souches de l'épithélium cornéen. Les cellules filles provenant de ces cellules souches se déplacent de façon centripète à partir du limbe, adoptant les caractéristiques de cellules amplificatrices transitoires. Quand, après plusieurs cycles de division cellulaire, leur contact diminue avec la membrane basale, elles se dirigent en surface, perdent leur capacité de prolifération et se différencient, amorçant l'expression des kératines $\mathrm{K} 3$ et K12 (Tableau I) avant de desquamer [4]. Certains des marqueurs proposés pour les cellules souches pourraient ne pas leur être exclusifs : ils pourraient, en effet, marquer également les cellules amplificatrices transitoires. Les cellules souches de la cornée seraient négatives pour les connexines 43 et 50 ( $C \times 43, C \times 50)$, protéines des jonctions gap, et positives pour la kératine $19[5,6]$, pour le facteur de transcription p63 [7] et pour $A B C G 2$ [8].

\section{Usages}

À l'extrême périphérie cornéenne, quelques cellules en gobelet sont observables (Figure 3 ). Ces cellules conjonctivales, qui sécrètent la composante muqueuse des larmes, sont absentes de la cornée normale. Lors de la destruction des cellules du limbe, les cellules conjonctivales migrent vers la cornée, suivies par une invasion vasculaire, un pannus et une perte de transparence. L'empreinte cornéenne montre alors des cellules positives pour la mucine (MUC5AC). L'architecture limbique disparaît; des déficits épithéliaux en progression sont accompagnés de cicatrisation, de kératinisation, de calcification ou d'inflammation chronique, voire d'ulcération et de perforation [4]. Ces altérations nuisibles pour l'acuité visuelle sont traitées en remplaçant ces cellules par des cellules limbiques provenant soit de l'œil controlatéral sain (autogreffe), soit de celui d'un donneur apparenté ou étranger (allogreffe). Le patient doit alors être immunodéprimé avec de la cyclosporine afin d'éviter le rejet.

Qu'elle soit acquise ou congénitale, la déficience en cellules souches (DCS) limbiques est à l'origine des problèmes cornéens de patients avec brûlures thermiques ou chimiques [1], de patients atteints de kératopathie induite par lentilles cornéennes [9], de pemphigoïde oculaire cicatriciel [2], d'aniridie [9] ou du syndrome de Stevens-Johnson [2]. 
Lorsque des cellules limbiques humaines sont mises en culture sur membrane amniotique, elles conservent les caractéristiques natives $[10,11]$ et présentent, par rapport à des cellules correspondantes en croissance sur du plastique, des proportions plus petites de cellules $\mathrm{K}^{+}$et $\mathrm{C} \times 43^{+}$et des proportions plus grandes de cellules positives pour le facteur de transcription p63 ou son isotype $\Delta \mathrm{Np} 63[10,11]$. L'amnios ralentit la différenciation des cellules limbiques et favorise le phénotype limbique; la croissance sur du plastique permet une transition vers un phénotype d'épithélium cornéen [10, 11].

Lors de la greffe in vivo, la membrane amniotique est transplantée et les cellules limbiques du receveur se multiplient au site de la greffe. Lorsque la DCS est complète, l'amplification en culture des cellules prélevées est souhaitable avant une greffe ex vivo. Un prélèvement de 2-3 mm de limbe sain produit, après culture, assez de cellules pour repeupler la cornée. Ces cellules peuvent être cultivées en absence ou en présence de cellules nourricières [1], permettant aux cellules limbiques de conserver un grand potentiel de prolifération et de régénération suggérant la conservation des cellules souches [6].
Des cellules limbiques et conjonctivales peuvent être mises en culture séparément sur une membrane amniotique avant d'être greffées à des patients avec brûlures oculaires. Comme la brûlure détruit les cellules limbiques et en gobelet, l'ajout de ces deux types cellulaires stabilise la surface oculaire et la prépare à l'éventuelle kératoplastie pénétrante qui suivra la reconstruction initiale [12]. De plus, l'amnios limite la formation d'adhérences du symblépharon, autre conséquence de la brûlure. II s'avère utile pour traiter une DCS congénitale comme l'aniridie [2].

L'amnios est également utilisé afin de traiter des affections des conjonctives bulbaires et palpébrales, des maladies cornéennes, en guise de tissu de remplacement pour les ulcères cornéens ou scléraux [2]. Il est indiqué pour traiter des défauts épithéliaux persistants dus à une kératopathie neurotrophique, à une kératite bulleuse ou d'exposition ou lors de kératoconjonctivite

\section{Intégrines \\ $\alpha 3 / \beta 1 \quad$ Récepteur de la membrane basale qui lie la laminine, la fibronectine et certains collagènes. Participe à la modulation de l'adhérence et de la migration cellulaires et dans l'organisation du cytosquelette des cellules épithéliales \\ $\alpha 6 / \beta 4 \quad$ Marqueur des hémidesmosomes reliant la cellule épithéliale à sa membrane basale. Marque donc la couche basale de l'épithélium}

\section{Kératines (K) et autre protéines}

\begin{tabular}{|c|c|c|c|c|}
\hline Marqueur & Marquage & $\begin{array}{l}\text { Épithélium } \\
\text { Conjonctival }\end{array}$ & Limbe & Épithélium cornéen \\
\hline K3 & Cellules différenciées & $\begin{array}{l}\text { Cellules superficielles } \\
\text { seulement }[27]\end{array}$ & $\begin{array}{l}\text { Cellules superficielles } \\
\text { seulement }\end{array}$ & $\begin{array}{l}\text { Toutes les couches au } \\
\text { centre }\end{array}$ \\
\hline $\mathrm{K} 12$ & Cellules différenciées & - & - & Épithélium exclusivement \\
\hline K19 & $\begin{array}{l}\text { Cellules souches de la cornée et/ } \\
\text { ou cellules amplificatrices transitoires }\end{array}$ & $+[28]$ & Cellules basales $+[5,6]$ & - \\
\hline MUC5AC & Mucine des cellules gobelets & + & - & - \\
\hline $\mathrm{Cx} 43$ & Connexine (jonctions gap) & & - & $+[8]$ \\
\hline $\begin{array}{l}\alpha-\varepsilon \text { nolase voir } \\
{[29]}\end{array}$ & Enzyme glycolytique & & $\begin{array}{l}\text { Toutes les cellules } \\
\text { basales et quelques } \\
\text { suprabasales }\end{array}$ & Cellules basales seules \\
\hline p63 $[7,30]$ & Nucléaire (facteur de transcription) & + & $\begin{array}{l}\text { Noyaux des cellules } \\
\text { basales, suprabasales }\end{array}$ & - \\
\hline Ki $67[31]$ & $\begin{array}{l}\text { Antigène nucléaire associé } \\
\text { à la prolifération }\end{array}$ & - & $\begin{array}{l}\text { Majorité des cellules } \\
\text { basales, suprabasales }\end{array}$ & - \\
\hline $\mathrm{ABCG} 2[8]$ & Marqueur postulé des cellules souches & & Cellules basales & - \\
\hline
\end{tabular}

Tableau I. Diverses protéines utiles au marquage des cellules épithéliales de la cornée et de la conjonctive normale (voir [26]). 
vernale atopique sévère et de pemphigoïde oculaire. L'emploi d'amnios chez des patients souffrant du syndrome de Stevens-Johnson a produit des résultats mitigés. Enfin, après un traumatisme, il sert parfois d'alternative à une lentille cornéenne pansement afin de réduire la friction de la paupière sur la surface épithéliale blessée lors du clignement [13].

\section{Propriétés}

La membrane amniotique contient de l'EGF (epidermal growth factor), du bFGF (basic fibroblast growth factor), du NGF (nerve growth factor) et d'autres facteurs de croissance. [14]. Or, l'ajout
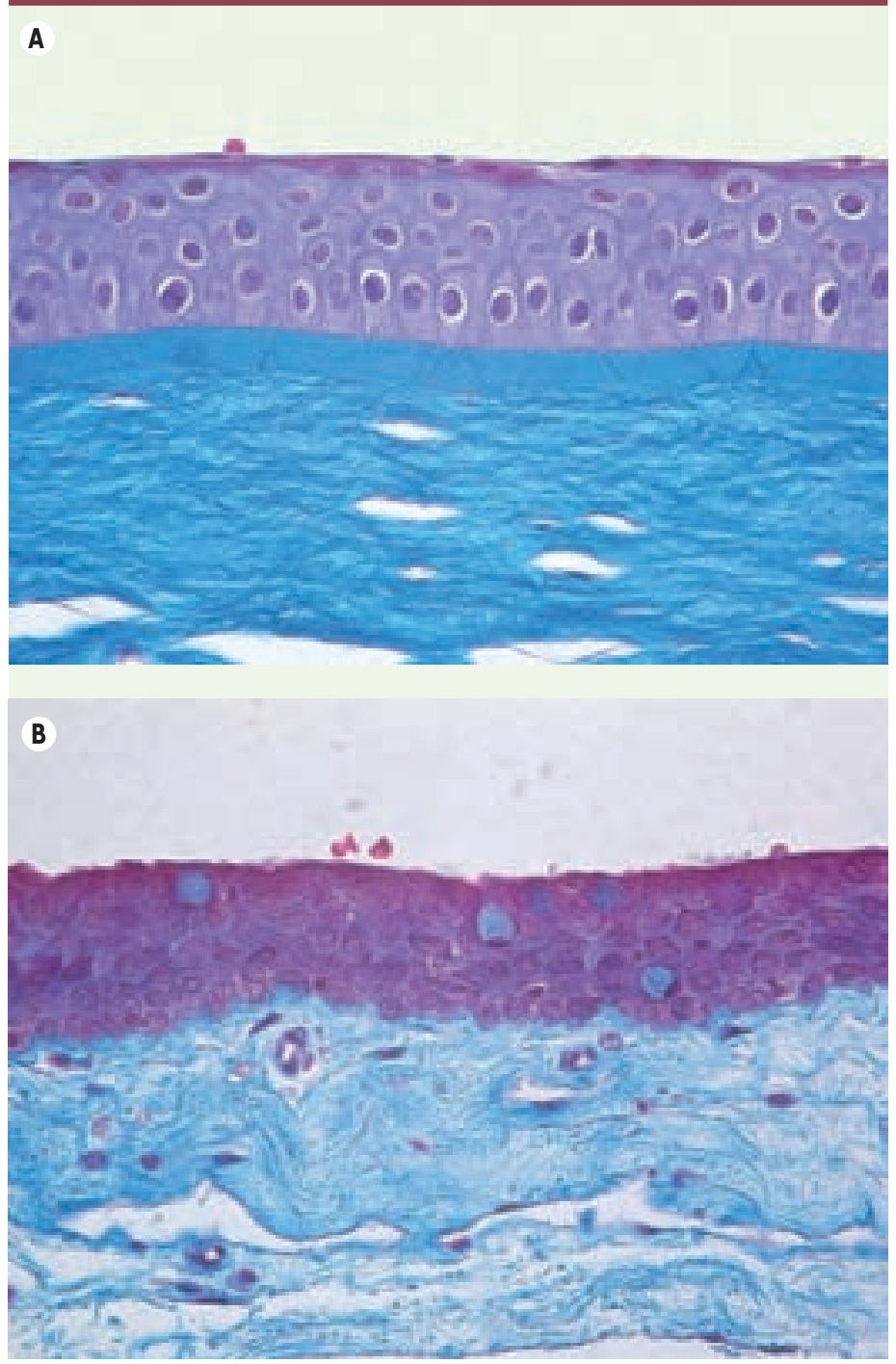

Figure 3. Section de paraffine illustrant les différences entre le centre (A) et la périphérie (B) de la cornée. En extrême périphérie, il est possible d’observer les cellules en gobelet de l'épithélium conjonctival (cellules bleutées au trichrome de Masson). Ces cellules sont absentes de la cornée normale, mais présentes dans la cornée qui se « conjonctivalise». de ces facteurs de croissance au milieu de culture favorise la prolifération des cellules épithéliales de la cornée et du limbe. La liaison à forte affinité du NGF avec son récepteur spécifique TrkA (tyrosine kinase-transducing receptor) des cellules basales de l'épithélium facilite et accélère la ré-épithélialisation. Ainsi, le NGF contenu dans l'amnios favorise la ré-épithélialisation de la cornée et facilite la guérison des ulcères neurotrophiques en permettant l'expression de récepteurs de NGF sur des cellules limbiques [15].

La membrane amniotique stimule l'antagoniste du récepteur de l'interleukine-1 (IL-l RA), une cytokine anti-inflammatoire et inhibe IL- $1 \alpha$ et $-1 \beta$, deux cytokines majeures de l'inflammation en abondance dans les larmes [16]. Elle peut contrer les effets de l'inflammation en inhibant des peptidases et en limitant l'entrée dans la cornée des neutrophiles lacrymaux et de leurs collagénases [17]. Au lendemain d'une chirurgie réfractive par laser excimer, la cornée d'un lapin couverte par une membrane amniotique compte moins de kératocytes en apoptose qu'une cornée témoin [18].

Les blessures d'étendue limitée guérissent sans former de cicatrice dans la peau fœtale. Cet effet est attribuable aux réactions différentes induites dans les fibroblastes fœtaux par des cytokines favorisant la formation de cicatrices dans leurs équivalents adultes [19] et à la présence de facteurs neurotrophiques dans l'amnios [17].

La reconstruction cornéenne ou conjonctivale par membrane amniotique réduit l'angiogenèse et la cicatrisation sur les surfaces. La culture sur membrane amniotique réduit l'expression de TGF- $\beta$ et de ses récepteurs dans des fibroblastes conjonctivaux ou cornéens. Or, la liaison de TGF- $\beta$ avec ses récepteurs est à l'origine de la fibrose [20]. Cette suppression de la voie de signalisation de TGF- $\beta$ par l'amnios est utile pour traiter les symblépharons dans lesquels des adhérences conjonctivo-cornéennes relient les paupières au globe oculaire.

La greffe de membrane amniotique procure une antalgie dans les kératopathies bulleuses en prévenant la formation douloureuse de bulles sous-épithéliales. Cette procédure s'avère une alternative acceptable à la kératoplastie pénétrante lorsque le potentiel visuel de l'œil douloureux est limité. La réponse immunologique consécutive à une transplantation de membrane amniotique est négligeable chez l'humain [1]. Enfin, l'amnios exerce des effets anti-bactériens par l'adhérence intime au substrat sous-jacent qui établit une barrière efficace contre l'infection [1]. 


\section{Limites}

La greffe de membrane amniotique a peu de succès en présence de perforation. Les cas d'ulcères profonds et de kératite neurotrophique exigent plus d'une intervention avant d'obtenir une acuité visuelle acceptable. Le tissu récupéré à la kératoplastie de patients porteurs d'une kératopathie neurotrophique montre une épithélialisation de la membrane amniotique et un tissu fibreux moins transparent qu'à l'origine. Cette résorption de la membrane amniotique semble se produire d'autant plus rapidement que la cornée est vascularisée, puisque ces vaisseaux fournissent des cellules inflammatoires et des peptidases en abondance [21].

Le traitement des défauts dans le stroma profond exige de faire appel à plus d'une couche de membrane amniotique: une seule couche de membrane disparaît après quelques semaines, soit avant qu'une synthèse suffisante de tissu ait pu combler l'espace laissé vacant par le défaut stromal [22]. La plupart des déficits épithéliaux persistants répondent bien aux traitements médicaux avec des gouttes lubrifiantes. L'utilisation de la membrane amniotique ne devrait donc pas remplacer ces traitements mais plutôt être une seconde option lorsque ceux-ci ne donnent pas les résultats escomptés [23]. Le taux d'échec est plus élevé quand l'amnios, utilisé comme pansement, dépasse le limbe et n'est pas suturé; l'inhibition de l'adhérence causée par la membrane amniotique entraîne le décollement de ce pansement en moins de deux semaines, à un moment où la guérison épithéliale est incomplète [24].

Il faudra déterminer si l'utilisation de la membrane amniotique offre, à long terme, de meilleurs rendements que les traitements traditionnels. L'hypothèse selon laquelle la membrane amniotique fournirait une niche favorable aux cellules souches résistera-t-elle à l'avènement de marqueurs propres à ces cellules? Les feuillets épithéliaux transplantés ont-ils une durée de vie comparable à celle du tissu normal ? En effet, il n'est pas démontré que les cellules épithéliales transplantées seront capables d'approvisionner en cellules l'épithélium cornéen pour le reste de la vie de l'individu.

\section{Contre-indications et complications possibles}

La détérioration de surface oculaire causée par un déficit lacrymal ou une kératinisation ne se prête pas à l'usage de membranes amniotiques [25]. Un tel recours peut entraîner chez le receveur les complications suivantes: pseudoptérygion, granulomes pyogéniques et calcification cornéenne. L'œil du donneur de cellules souches peut subir une déficience grave de ces cellules attribuable à leur prélèvement ou encore une kératite filamenteuse, un pseudoptérygion et des défauts épithéliaux. C'est pourquoi, le prélèvement d'un minimum de cellules limbiques amplifiées en culture diminue les risques de complications dans I'œil du donneur.

\section{Conclusions}

Les propriétés de la membrane amniotique ouvrent des perspectives intéressantes dans les traitements de troubles oculaires graves et permettent d'espérer une meilleure qualité de vie pour les greffés. Est-il si surprenant que cette membrane, qui renferme les premières cellules d'une vie naissante, bénéficie de propriétés telles qu'à son contact, la régénération d'une population cellulaire soit favorisée? Probablement pas. La découverte des mécanismes à l'origine de cette stimulation pourrait offrir des traitements nouveaux (certes encore à mettre au point) qui, en exploitant les interactions entre la membrane amniotique et les cellules souches, répareraient les tissus avec un minimum de cicatrices, de douleurs ou de complications. De plus, les connaissances ainsi acquises pourraient conduire à l'amélioration des techniques de reconstruction de la cornée par génie tissulaire. L'une des méthodes prometteuses est appelée la méthode d'auto-assemblage car, sous certaines conditions contrôlées, les fibroblastes cornéens synthétisent une matrice qu'il est possible d'utiliser dans la fabrication d'un analogue du stroma cornéen. II suffit ensuite d'ajouter sur cette matrice extracellulaire les cellules épithéliales et endothéliales afin de reconstituer un analogue de la cornée complète [26].

II reste à approfondir les effets sur l'œil du tissu amniotique et à espérer que d'ici peu, les secrets qu'il recèle encore pourront être dissipés et utilisés directement ou appliqués au développement de substituts cornéens produits par génie tissulaire afin de traiter un bon nombre d'affections oculaires et de réduire la souffrance humaine qui en est la conséquence. $\diamond$

\section{SUMMARY}

\section{Amnion and ocular surface problems}

The amniotic membrane, the most internal placental membrane, has various properties useful in ophthalmology. Collected on delivery by elective Caesarean section, the amnion is prepared under sterile conditions, and, usually, cryopreserved until its use as a biological bandage or as a substrate for epithelial growth in the management of various ocular surface conditions. Specifically, the amnion is used to : (1) limit formation of adhesive bands between eyelids and eyeball (symblepharon) or the progression of a fibrovascular outgrowth towards the cornea (pterygium) or to (2) facilitate the healing of corneal ulcers, bullous keratopathy, and corneal stem cell deficiency. In this last condition, either hereditary or acquired after a thermal or a chemical burn, corneal stem cells, located at a transitional zone between the cornea and conjunctiva, are lost. These cells are essential for renewal of corneal epithelium in normal and in diseased states. The loss of these cells leaves the corneal surface free for invasion by conjunctival epithelium. Not only, does conjunctival epithelium support the 
development of vascularisation on the normally avascular cornea, but some conjunctival cells differentiate into mucus secreting goblet cells. Such a change in phenotype leads to loss of corneal transparency and visual disability. The removal of this fibro-vascular outgrowth in combination with transplantation of both amniotic membrane and corneal stem cells are used to treat this condition. The amnion stimulates the proliferation of less differentiated cells which have the potential to reconstruct the cornea. This potential is at the origin of the hypothesis that the amnion may provide an alternative niche for limbal stem cells of the corneal epithelium. It abounds in cytokines and has antalgic, anti-bacterial, anti-inflammatory and anti-immunogenic properties, in addition to allowing, like fetal skin does, wound healing with minimal scar formation. These desirable properties are responsible for the increasing use of amniotic membrane in ophthalmology. The complete understanding of the mechanisms of action of amniotic membrane for ocular surface diseases has yet to be understood. Once revealed by research, they may provide new pharmacological avenues to treat ocular surface diseases. $\diamond$

\section{RÉFÉRENCES}

1. Dua HS, Gomes JA, King AJ, Maharajan VS. The amniotic membrane in ophthalmology. Surv Ophthalmol $2004 ; 49$ : 51-77.

2. Bouchard CS, John T. Amniotic membrane transplantation in the management of severe ocular surface disease : indications and outcomes. The Ocular Surface 2004 ; 2 : 201-11.

3. John T. Human amniotic membrane transplantation : past, present, and future. Ophthalmol Clin North Am 2003; $16: 43-65$

4. Dua HS, Joseph A, Shanmuganathan VA, Jones RE. Stem cell differentiation and the effects of deficiency. Eye $2003 ; 17: 877-85$.

5. Kim HS, Jun Song X, de Paiva CS, et al. Phenotypic characterization of human corneal epithelial cells expanded ex vivo from limbal explant and single cell cultures. Exp Eye Res $2004 ; 79: 41-9$.

6. Lindberg K, Brown ME, Chaves HV, et al. In vitro propagation of human ocular surface epithelial cells for transplantation. Invest Ophthalmol Vis Sci $1993 ; 34: 2672-9$.

7. Di lorio $\varepsilon$, Barbaro V, Ruzza A, et al. Isoforms of DeltaNp63 and the migration of ocular limbal cells in human corneal regeneration. Proc Natl Acad Sci USA 2005 ; 102 : 9523-8.

8. Wolosin JM, Budak MT, Akinci MA. Ocular surface epithelial and stem cell development. Int J Dev Biol 2004 ; 48 : 981-91.

9. Tseng SC, Prabhasawat P, Barton K, et al. Amniotic membrane transplantation with or without limbal allografts for corneal surface reconstruction in patients with limbal stem cell deficiency. Arch Ophthalmol 1998; 116: 431-41.

10. Hernandez Galindo દદ, Theiss C, Steuhl KP, Meller D. Expression of Delta Np63 in response to phorbol ester in human limbal epithelial cells expanded on intact human amniotic membrane. Invest Ophthalmol Vis Sci $2003 ; 44: 2959-65$.

11. Du Y, Chen J, Funderburgh JL, et al. Functional reconstruction of rabbit corneal epithelium by human limbal cells cultured on amniotic membrane. Mol Vis 2003; 9:635-43.

12. Sangwan VS, Vemuganti GK, Singh S, Balasubramanian D. Successful reconstruction of damaged ocular outer surface in humans using limbal and conjuctival stem cell culture methods. Biosci Rep $2003 ; 23: 169-74$.

13. Baum J. Thygeson lecture. Amniotic membrane transplantation : why is it effective? Cornea $2002 ; 21: 339-41$.
14. Koizumi N, Inatomi TJ, Sotozono C, et al. Growth factor mRNA and protein in preserved human amniotic membrane. Curr Eye Res $2000 ; 20: 173-7$.

15. Touhami A, Grueterich M, Tseng SC. The role of NGF signaling in human limbal epithelium expanded by amniotic membrane culture. Invest Ophthalmol Vis Sci $2002 ; 43: 987-94$.

16. Solomon A, Rosenblatt M, Monroy D, et al. Suppression of interleukin lalpha and interleukin lbeta in human limbal epithelial cells cultured on the amniotic membrane stromal matrix. Br J Ophthalmol 2001 ; $85: 444-9$.

17. Tseng SC, Espana EM, Kawakita T, et al. How does amniotic membrane work? The Ocular Surface $2004 ; 2: 177-87$.

18. Park WC, Tseng SC. Modulation of acute inflammation and keratocyte death by suturing, blood, and amniotic membrane in PRK. Invest Ophthalmol Vis Sci $2000 ; 41: 2906-14$.

19. Moulin V, Tam BY, Castilloux G, et al. Fetal and adult human skin fibroblasts display intrinsic differences in contractile capacity. J Cell Physiol 2001; 188:211-22.

20. Lee SB, Li DQ, Tan DT, et al. Suppression of TGF-beta signaling in both normal conjunctival fibroblasts and pterygial body fibroblasts by amniotic membrane. Curr Eye Res $2000 ; 20$ : 325-34.

21. Gris 0 , Wolley-Dod C, Guell JL, et al. Histologic findings after amniotic membrane graft in the human cornea. Ophthalmology 2002 ; $109: 508-12$.

22. Koizumi N, Inatomi T, Quantock AJ, et al. Amniotic membrane as a substrate for cultivating limbal corneal epithelial cells for autologous transplantation in rabbits. Cornea $2000 ; 19: 65-71$.

23. Gris 0 , Campo Z, Wolley-Dod C, et al. Amniotic membrane implantation as a therapeutic contact lens for the treatment of epithelial disorders. Cornea $2002 ; 21: 22-7$.

24. Muraine $M$, Descargues $G$, Franck 0 , et al. La greffe de membrane amniotique dans les pathologies oculaires de surface. Étude prospective à partir de 31 cas. J Fr Ophtalmol $2001 ; 24: 798-812$.

25. Tseng SC. Amniotic membrane transplantation for ocular surface reconstruction. Biosci Rep 2001 ; 21 : 481-9.

26. Germain L, Giasson CJ, Carrier P, et al. Tissue engineering of cornea. In : Wnek GE, Bowlin GL, eds. Encyclopedia of biomaterials and biomedical engineering. New York: Marcel Dekker, 2004 : 1534-44.

27. Nakamura $T$, Nishida K, Dota A, et al. Elevated expression of transglutaminase $l$ and keratinization-related proteins in conjunctiva in severe ocular surface disease. Invest Ophthalmol Vis Sci 2001 ; $42: 549-56$.

28. Deschambeault A, Carrier P, Talbot M, Germain L. In vitro Characterization of human limbal epithelial cells isolated from the four quadrants. ARVO Meeting Abstracts 2003; $44: 1357$.

29. Lavker RM, Tseng SC, Sun TT. Corneal epithelial stem cells at the limbus: looking at some old problems from a new angle. Exp Eye Res 2004 ; $78: 433-46$.

30. Sakoonwatanyoo P, Tan DT, Smith DR. Expression of $p 63$ in pterygium and normal conjunctiva. Cornea $2004 ; 23: 67-70$.

31. Abu El-Asrar AM, Al-Mansouri S, Tabbara KF, et al. Immunopathogenesis of conjunctival remodelling in vernal keratoconjunctivitis. Eye 2006 ; $20: 71-9$.

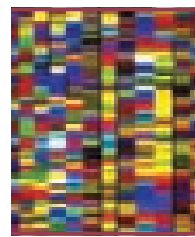

Retrouvez chaque mois médecine/sciences sur France-Info dans la chronique «Info-Sciences 》 de Marie-Odile Monchicourt, du lundi au mercredi. france-info.com 